\title{
BMJ Open Effect of backward walking training on knee osteoarthritis: protocol of a systematic review and meta-analysis
}

\author{
Yuxuan Wu, ${ }^{1}$ Cheng Lei (D) , ${ }^{1}$ Zhimin Huangfu, ${ }^{2}$ Kejimu Sunzi, ${ }^{1}$ Changmei Yang ${ }^{3}$
}

To cite: Wu Y, Lei C, Huangfu Z, et al. Effect of backward walking training on knee osteoarthritis: protocol of a systematic review and meta-analysis. BMJ Open 2020;10:e040726. doi:10.1136/ bmjopen-2020-040726

- Prepublication history for this paper is available online. To view these files, please visit the journal online (http://dx.doi org/10.1136/bmjopen-2020040726).

Received 21 May 2020 Revised 17 September 2020 Accepted 07 October 2020
Check for updates

(C) Author(s) (or their employer(s)) 2020. Re-use permitted under CC BY-NC. No commercial re-use. See rights and permissions. Published by BMJ.

${ }^{1}$ Department of Orthopedics, People's Hospital of Deyang City, Deyang, China

${ }^{2}$ College of Basic Medical Sciences, Chongqing Medical University, Chongqing, China ${ }^{3}$ Department of Neurosurgery, The Affiliated Hospital of Southwest Medical University, Luzhou, China

Correspondence to Cheng Lei; lifecool_Ic@163.com and Professor Changmei Yang; 2081322034@qq.com

\section{ABSTRACT}

Introduction Backward walking (BW) is otherwise known as retrowalking. As opposed to forward walking, BW is a countersequential exercise and is a common method of rehabilitation training and disease-assisted treatment. Studies have shown that BW has a helpful effect on improving lower limb proprioception, gait synergy and improving limb balance. Many studies have concluded that BW can improve the symptoms of patients with knee osteoarthritis (KOA) and can be used for rehabilitation and adjunctive treatment of KOA, but there is a lack of evidence-based medical evidence. This research aims to provide an update to the most recent available evidence on the effect of BW on patients with KOA.

Methods and analyses Electronic databases, such as Ovid/MEDLINE, EMBASE, CINAHL, Scopus, Web of Science and PubMed, will be searched by us. We will include studies identified from citation until 12 May 2020 and will not be restricted by geographical setting. The search will not be limited to the language of the publication, but the study of human subjects. Randomised controlled trials (RCTs) on the BW training of KOA will be included, with outcome measures including pain, knee function or balance function. The quality of included RCTs will be evaluated according to the Cochrane Collaboration's risk of bias tool. A meta-analysis or systematic review will be performed to summarise the effects of BW training. We will perform sensitivity analysis on the sample size of RCTs, meta-regression analysis of the follow-up periods, dosages and baselines of outcome measures, and publication bias analysis.

Ethics and dissemination Ethical approval is not required as this study will not involve confidential personal data. The results of this study will be disseminated through a peer-reviewed journal.

\section{PROSPERO registration number CRD42020185694.}

\section{INTRODUCTION}

Osteoarthritis (OA) is a common chronic disease that causes disability in the elderly, affecting the joints of the hands, hips, knees and spine, with knee OA (KOA) being the most common. ${ }^{1}$ In China, the prevalence of $\mathrm{KOA}$ is about $18 \%$, and patients with $\mathrm{KOA}$ often experience pain, decreased muscle mass and function, impaired proprioception and body imbalance, all of which can reduce postural stability and increase the risk of falls,

\section{Strengths and limitations of this study}

This review will provide a comprehensive, standardised and extensive search strategy.

- This review will compare the effects of backward walking (BW) training and other traditional sports rehabilitation training on patients with knee osteoarthritis (KOA)

- This review will provide a comprehensive assessment regarding the effect of $\mathrm{BW}$ training in patients with KOA.

- The results will help patients with KOA to have more options for rehabilitation training.

- The comprehensiveness and methodological quality of the main studies included in this review may be poor, leading to a significant discount in the reliability of the results.

which places a huge burden on patients and their caregivers. ${ }^{2-4}$ Currently, pharmacological interventions, joint cavity injections and surgery are mostly used to alleviate the clinical symptoms of KOA. ${ }^{5}$ With the emphasis on healthy lifestyles, an increasing number of people are opting for exercise interventions to reverse the progression or perform postoperative rehabilitation exercises. ${ }^{78}$

Backward walking (BW) is a universal method of rehabilitation training and adjuvant therapy. ${ }^{9}$ It is confirmed that BW can reduce the symptoms of female patients with patellofemoral pain syndrome by affecting the activity rate of medial femoral oblique and lateral femoral muscle. ${ }^{10}$ In addition, BW has a beneficial effect on improving lower limb proprioception, gait synergy and limb balance. ${ }^{11-14}$ Results of a study recommend the use of BW for KOA rehabilitation and adjuvant therapy, because they found that BW can reduce the pain caused by arthritis, optimise joint function and enhance the strength of the quadriceps muscles. ${ }^{15}$ However, it has been reported that while walking rehabilitation therapy can improve cardiovascular health in patients with KOA, 
it does not make any significant effect on reducing pain symptoms. ${ }^{16}$

At present, most researchers believe that BW training may improve the symptoms of patients with KOA and can be used to rehabilitation and adjuvant therapy, but there is still no research to summarise the evidence. Therefore, this study will collect randomised controlled trials (RCTs) in which BW training is used for the rehabilitation and adjuvant treatment of patients with $\mathrm{KOA}$, and conduct a systematic review and meta-analysis to clarify the effect of BW training in atients with KOA.

\section{Objective}

The main purpose of this study is to determine the rehabilitation effect of BW training on KOA. We aim to conduct a systematic review and meta-analysis of RCTs to compare BW training with any other rehabilitation therapy (eg, walking forward, manual massage, traditional exercise therapy, etc). The main outcomes are inflammation relief, joint function recovery and adverse events.

\section{METHODS}

This protocol will be conducted in accordance with the guideline of Preferred Reporting Items for Systematic Reviews and Meta-Analyses Protocols (PRISMA) 2015 statement. ${ }^{17}$

\section{ELIGIBILITY CRITERIA \\ Participants}

Adults (over 18 years old) suffering from KOA will be the participants in our study. We will not restrict patients from different clinical staging. Age, sex or ethnicity of the enrolled subjects will not be the restriction.

\section{Interventions}

The mode of intervention is BW as rehabilitation training with any route, dose or frequency. We also will include trials where BW training is used as an adjunctive therapy to conservative treatments.

\section{Comparators}

The comparators are any other rehabilitation therapy (eg, walking forward, manual massage, traditional exercise therapy, etc), or no intervention.

\section{Outcomes}

The outcomes will include (1) pain perception, (2) knee function recovery, (3) mobility, (4) improvement movable degree of knee joint, (5) quadriceps strength and (6) adverse events.

\section{Study characteristics}

We decide to include RCTs which are more likely to provide unbiased information than other study designs. However, quasi-randomised RCTs (eg, allocation by alphabetical order, medical record number, alternate days of the week or date of birth) and cross-over trials will also be included. There is no restriction on language or publication status.

We will exclude those that do not have a control group design, do not provide case and control sources, reviews, case or expert experience, animal experiments, nursing records, conference proceedings, repeated publications, incomplete data and collection of unscientific literature.

\section{INFORMATION SOURCES}

We will conduct electronic searches through these databases: Ovid/MEDLINE, EMBASE, CINAHL, Scopus, Web of Science and PubMed. The search period ended on 12 May 2020. We will also conduct manual searches of journal volumes/journals and conference proceedings that are not available in electronic search to review references. And contact the author for the full text of the identified literature. We will carry out a 'snowballing'search to find relevant studies from the reference list of included studies and previous systematic reviews. In addition, we will also contact subject experts and authors of the identified research/organisations to obtain relevant researches for inclusion in our review.

\section{Search strategy}

Search strategies will be developed using medical subject headings as well as text words associated with terms relevant to 'backward walking', 'knee osteoarthritis' together with 'randomised controlled trial'. The searches will be conducted by two authors independently (YW and CL). Table 1 lists an example of our search strategy.

\section{Study records}

\section{Data management}

EndNote V.X7 will be used to manage literature search results, and remove duplicate records. All extracted data are stored in a Microsoft Excel spread sheet.

\section{Selection process}

The PRISMA-compliant flow chart will be used to demonstrate the literature selection process (figure 1), all conducted by two authors (ZH and KS), while the third author (CY) can help resolve any differences. First, the titles and abstracts of all retrieved articles will be reviewed independently, and studies that clearly do not meet the eligibility criteria will be excluded. Then, to determine if they meet the eligibility criteria, each author will further review the full text of the remaining studies. The results will be collated if multiple reports are found in the same study.

\section{Data collection process}

Data for all eligible publications will be carefully and independently extracted by two authors (YW and CL) in two copies. Disagreements encountered in the process will be resolved through discussion and, if necessary, can be resolved with the help of a third author (CY). Additional attachments will be sought or study authors will be contacted via email to obtain raw data as well as missing 


\begin{tabular}{|c|c|}
\hline No & Search items \\
\hline \#1 & Randomized controlled trial.pt \\
\hline \#2 & Controlled clinical trial.pt \\
\hline \#3 & Randomized.ti,ab \\
\hline \#4 & Randomly.ti,ab \\
\hline \#5 & Trial.ti,ab \\
\hline \#6 & Placebo.ti,ab \\
\hline \#7 & Groups.ti,ab \\
\hline \#8 & $\# 1$ or \#2 or \#3 or \#4 or \#5 or \#6 or \# \\
\hline \#8 & Knee Osteoarthritis.Mesh \\
\hline$\# 9$ & Knee Joint.Mesh \\
\hline \#10 & Patellofemoral Joint.Mesh \\
\hline \#11 & \#8 or \#9 or \#10 \\
\hline \#12 & backward.ti,ab \\
\hline \#13 & Retro.ti,ab \\
\hline \#14 & \#12 or \#13 \\
\hline \#15 & walking.ti,ab \\
\hline \#16 & gait.ti,ab \\
\hline \#17 & locomotion.ti,ab \\
\hline \#18 & $\# 15$ or \#16 or \#17 \\
\hline \#19 & $\# 14$ and \#18 \\
\hline \#20 & $\# 8$ and \#11 and \#19 \\
\hline
\end{tabular}

data. After sending two request emails within 4 weeks with no response, we will drop the contact.

\section{Data items}

The following information will be extracted using a predetermined data form from each included study: author, year of publication, country of study, sample size, age, sex, disease status, setting, intervention type, intervention

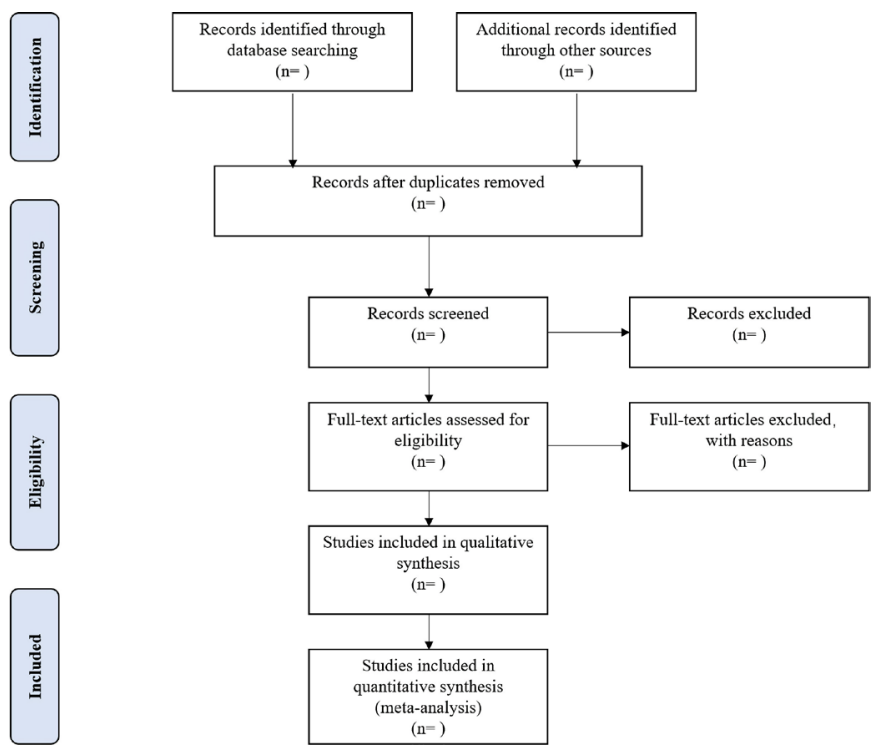

Figure 1 The primary selection process. dose, follow-up duration, intervention components, measures, key outcomes, other outcomes and estimated intervention effectiveness.

\section{Outcomes and prioritisation}

Since soothed knee inflammation and joint function recovery are key outcome indicators after rehabilitation training, the main outcomes of this study will be reduced pain perception and joint function recovery in patients. Reduced pain perception can be assessed through patient self-report questionnaires, such as the Numeric Rating Scale for Pain ${ }^{15}$; joint functional recovery is defined as improved mobility and will be assessed through scales and tests, such as the Timed ' $U p$ and Go' test ${ }^{15}$ or the Western Ontario and McMaster Universities Osteoarthritis Index. $^{15} 18$

The secondary outcomes will assess adverse events that occurred during the intervention or during follow-up, including dizziness, anxiety, falls and other adverse events related to exercise training. ${ }^{19}$

\section{Risk of bias in individual studies}

The risk of bias for each included study will be independently assessed by two reviewers (YW and CL). If there is a disagreement, it will be resolved through discussion or the decision of the third author (CY). The PEDro scale was used to analyse the risk of bias for the included trials. ${ }^{20}$ The scale included 11 items: (1) eligibility criteria were specified, (2) subjects were randomly allocated to groups (in a cross-over study, subjects were randomly allocated an order in which treatments were received), (3) allocation was concealed, (4) the groups were similar at baseline regarding the most important prognostic indicators, (5) there was blindingof all subjects, (6) there was blinding of all therapists who administered the therapy, (7) there was blinding of all assessors who measured at least one key outcome, (8) measures of at least one key outcome were obtained from more than $85 \%$ of the subjects initially allocated to groups, (9) all subjects for whom outcome measures were available received the treatment or control condition as allocated or, where this was not the case, data for at least one key outcome was analysed by 'intention to treat', (10) the results of between-group statistical comparisons are reported for at least one key outcome and (11) the study provides both point measures and measures of variability for at least one key outcome.

\section{Data synthesis}

The main outcome indicators involved in this study are mostly continuous variables, a standardised mean difference will be used to express the result. Adverse events will be descriptively analysed, if applicable (for dichotomous outcomes) a risk ratio with $95 \% \mathrm{Cl}$ will be reported. For those who do not clearly present for the data or change values in the literature, we will obtain the original data by contacting the original author. If the contact is unsuccessful, the relevant evidence-based data 
calculation formula will be used. The pooled data will be conduct through Stata V.12.0 (StataCorp) with the metan command.

The heterogeneity included in the research results will be analysed by $\chi^{2}$ test, and the effect model will be selected according to the analysis results. If $\mathrm{p} \geq 0.1$ and $\mathrm{I}^{2}<50 \%$, it indicates that the statistical heterogeneity between the studies is small, and the fixed-effect model can be selected for data combination; if $\mathrm{p}<0.1, \mathrm{I}^{2} \geq 50 \%$, it indicates that there may be obvious heterogeneity between the studies then we will choose a random effects model for data merging. ${ }^{21}$ When sufficient data are available, we will perform a subgroup analysis to investigate heterogeneity.

We will perform subgroup analysis based on age, gender, training dose, follow-up time and control group (forward walking training, no treatment or other therapies). In addition, sensitivity analyses will be conducted to examine the robustness of our analysis by omitting specific trials from the overall analysis.

If quantitative synthesis is not applicable, we will perform narrative, qualitative summaries and use sequential text and tables to present the information.

\section{Meta-bias}

If 10 or more studies are included in the meta-analysis, a funnel plot will be used to qualify the study effects and the Egger's test will be used for qualitative analysis. ${ }^{22}$ Reporting bias, if any, will be assessed by comparing the study results with its protocol.

\section{Confidence in cumulative evidence}

We will use the Grading of Recommendations Assessment, Development and Evaluation approach to assess the quality of each summarised evidence. The risk of bias, consistency, directness, imprecision and publication bias are the main evaluation indicators of this method. The overall quality of evidence will be rated as high, moderate, low or very low. The evidence evaluation process will be conducted using the GRADEpro online software (http:// gradepro.org) ${ }^{23}$

\section{Amendments}

If there is any modification of the agreement, we will provide the date of modification, explanation and reason for the event of protocol amendments.

Contributors YW and CL contributed to the conception and design of this study protocol. CL registered the protocol with the PROSPERO database. YW and CL edited the draft protocol. The search strategy was developed by $\mathrm{CL}$ and YW. ZH and KS will screen titles, abstracts and full-text copies of the studies after the literature search. YW, CL or ZH will extract information of the included studies after screening. $\mathrm{KS}$ and YW will check the data entry for accuracy and completeness. $\mathrm{CY}$ is the guarantor for this review and will give advice for data analysis and presentation of study result. All the authors drafted and critically reviewed this manuscript and approved the final version.

Funding This study was supported by Scientific Research Project of Sichuan Provincial Health and Family Planning Commission of China (17ZD023) and Science and Technology Plan Projects of Deyang City (2018SZS091).

Competing interests None declared.
Patient and public involvement Patients and/or the public were not involved in the design, or conduct, or reporting, or dissemination plans of this research.

Patient consent for publication Not required.

Provenance and peer review Not commissioned; externally peer reviewed.

Open access This is an open access article distributed in accordance with the Creative Commons Attribution Non Commercial (CC BY-NC 4.0) license, which permits others to distribute, remix, adapt, build upon this work non-commercially, and license their derivative works on different terms, provided the original work is properly cited, appropriate credit is given, any changes made indicated, and the use is non-commercial. See: http://creativecommons.org/licenses/by-nc/4.0/.

ORCID iD

Cheng Lei http://orcid.org/0000-0003-1311-515X

\section{REFERENCES}

1 Hunter DJ, Bierma-Zeinstra S. Osteoarthritis. Lancet 2019;393:1745-59.

2 Bin W, Dan X, Shengjie D, et al. Prevalence and disease burden of knee osteoarthritis in China: a systematic review. Chinese Journal of Evidence-Based Medicine 2018;18:134-42.

3 de Rooij M, van der Leeden M, Heymans MW, et al. Prognosis of pain and physical functioning in patients with knee osteoarthritis: a systematic review and meta-analysis. Arthritis Care Res 2016;68:481-92.

4 Manlapaz DG, Sole G, Jayakaran P, et al. Risk factors for falls in adults with knee osteoarthritis: a systematic review. $P m R$ 2019;11:745-57.

5 Rodriguez-Merchan EC, Merchan R, Carlos E. Conservative treatment of acute knee osteoarthritis: a review of the Cochrane library. Journal of Acute Disease 2016;5:190-3.

6 Tian $\mathrm{K}$, Cheng $\mathrm{H}$, Zhang J, et al. Intra-Articular injection of methylprednisolone for reducing pain in knee osteoarthritis: a systematic review and meta-analysis. Medicine 2018;97: 0240

7 Fransen M, McConnell S, Harmer AR, et al. Exercise for osteoarthritis of the knee: a Cochrane systematic review. Br J Sports Med 2015;49:1554-7.

8 Timmins KA, Leech RD, Batt ME, et al. Running and knee osteoarthritis: a systematic review and meta-analysis. Am J Sports Med 2017:45:1447-57.

9 Balasukumaran T, Olivier B, Ntsiea MV. The effectiveness of backward walking as a treatment for people with gait impairments: a systematic review and meta-analysis. Clin Rehabil 2019;33:171-82.

10 Abdelraouf OR, Abdel-Aziem AA, Ahmed AA, et al. Backward walking alters vastus medialis oblique/vastus lateralis muscle activity ratio in females with patellofemoral pain syndrome. Turk J Phys Med Rehabil 2019;65:169-76.

11 Wang J, Yuan W, An R. Effectiveness of backward walking training on spatial-temporal gait characteristics: a systematic review and meta-analysis. Hum Mov Sci 2018;60:57-71.

12 Elnahhas AM, Elshennawy S, Aly MG. Effects of backward gait training on balance, gross motor function, and gait in children with cerebral palsy: a systematic review. Clin Rehabil 2019;33:3-12.

13 Groff BR, Antonellis P, Schmid KK, et al. Stride-time variability is related to sensorimotor cortical activation during forward and backward walking. Neurosci Lett 2019;692:150-8.

14 Rose DK, DeMark L, Fox EJ, et al. A backward walking training program to improve balance and mobility in acute stroke: a pilot randomized controlled trial. J Neurol Phys Ther 2018;42:12-21.

15 Alghadir AH, Anwer S, Sarkar B, et al. Effect of 6-week retro or forward walking program on pain, functional disability, quadriceps muscle strength, and performance in individuals with knee osteoarthritis: a randomized controlled trial (retro-walking trial). BMC Musculoskelet Disord 2019;20:159.

16 Wallis JA, Webster KE, Levinger $\mathrm{P}$, et al. A walking program for people with severe knee osteoarthritis did not reduce pain but may have benefits for cardiovascular health: a phase II randomised controlled trial. Osteoarthritis Cartilage 2017;25:1969-79.

17 Moher D, Shamseer L, Clarke M, et al. Preferred reporting items for systematic review and meta-analysis protocols (PRISMA-P) 2015 statement. Syst Rev 2015;4:1.

18 Gondhalekar GA, Deo MV. Retrowalking as an adjunct to conventional treatment versus conventional treatment alone on pain and disability in patients with acute exacerbation of chronic knee osteoarthritis: a randomized clinical trial. N Am J Med Sci 2013;5:108-12. 
19 Lei C, Sunzi K, Dai F, et al. Effects of virtual reality rehabilitation training on gait and balance in patients with Parkinson's disease: a systematic review. PLoS One 2019;14:e0224819.

20 Beaton DE, Bombardier C, Guillemin F, et al. Recommendations for the cross-cultural adaptation of health status measures. Available: http://www. dash. iwh. on. ca/assets/images/pdfs/xculture2002. pdf [Accessed 1 Oct 2009].
21 Delgado-Rodríguez M, Sillero-Arenas M. Systematic review and meta-analysis. Med Intensiva 2018;42:444-53.

22 Jin Z-C, Zhou X-H, He J. Statistical methods for dealing with publication bias in meta-analysis. Stat Med 2015;34:343-60.

23 Guyatt GH, Oxman AD, Schünemann $\mathrm{HJ}$, et al. GRADE guidelines: a new series of articles in the Journal of clinical epidemiology. J Clin Epidemiol 2011;64:380-2. 